\title{
LITERATURE REVIEW: EFFECT OF EXERCISE ON SERUM FGF23
}

\author{
Nurunnisa Sholeha Letto , Vita Murniati Tarawan², Hanna Goenawan², Yuni Susanti Pratiwi², Titing \\ Nurhayati $^{2}$ \\ ${ }^{1}$ Faculty of Medicine, Universitas Padjadjaran, Bandung, Indonesia \\ ${ }^{2}$ Physiology Division, Department of Basic Medical Science, Faculty of Medicine, Universitas Padjadjaran, Bandung, \\ Indonesia
}

\begin{abstract}
Exercise is an activity that has many health benefits, including bone, heart, and muscle health. One of the proteins found to be increased after exercise was FGF23. FGF23 is one of the bone mineral regulators which previously served as an indicator for chronic kidney disease severity and cardiovascular morbidity. However, some studies have found no increase in FGF23 after exercise. The purpose of this study is to elucidate the effect of exercise on serum FGF23. This study is a literature review with a qualitative approach comparing previous studies related to exercise and FGF23. From 13 selected literatures, serum FGF23 elevation was found on exercise for one day to three weeks and did not change after six weeks to 12 months of exercise. The conclusions of the study are serum FGF23 was found to be increased after one day to three weeks of exercise and serum FGF23 in human studies found to be increased after long-duration maximal-intensity exercise.
\end{abstract}

Keywords: exercise, FGF23, FITT, metabolic adaptation 


\section{INTRODUCTION}

Exercise is defined by WHO as a subcategory of physical activity that is purposefully done to gain physical fitness. ${ }^{1}$ Exercise has been known to have numerous benefits for health. It is proved to improve cardiorespiratory, bone, and muscular fitness. It is well known to reduce the risk of non-communicable diseases, such as conditions in metabolic syndrome. ${ }^{1-3}$ These health benefits of exercise led to the discovery of factors that could improve exercise performance. In 2016, Li et al. found that Fibroblast Growth Factor-23 (FGF23) treatment on exercise associated with lower ROS, increased mitochondrial function, and increased time to exhaustion. Therefore, they suggested that FGF23 could improve exercise performance. ${ }^{4}$

FGF23 is a member of the FGF protein family, which is a family of proteins that shared structural similarity. ${ }^{5}$ The FGF family has been classified into seven subclasses, one of them is the FGF19 subclass (consist of FGF19, FGF21, FGF23) which is also referred to as endocrine FGF or metabolic FGF. ${ }^{5,6}$ FGF23 produced primarily by osteocytes. $^{7}$ FGF23 main functions is to regulate phosphorus and vitamin D metabolism. ${ }^{7}$ FGF23 acts mainly in renal tubules and further became a biomarker for renal function, especially in chronic kidney disease (CKD) where it indicates the adverse outcome of CKD. ${ }^{7-9}$ FGF23 also has direct activity on cardiac muscle and inflammation, where elevated FGF23 concentration is associated with left ventricle hypertrophy greater risk of cardiovascular morbidity. ${ }^{8-11}$

The importance of FGF23 as a biomarker of CKD and cardiovascular disease (CVD) caused this protein to be commonly studied in clinical settings. Studies about FGF23 and exercise was initially carried out regarding the function of FGF23 as a bone mineral regulator. The study showed that exercise increase serum FGF23. ${ }^{12}$ Subsequently, studies related to FGF23 with exercise began to develop with various exercise modalities. ${ }^{13}$

However, the findings are still inconsistent whether exercise increases FGF23 or not. ${ }^{4,14}$ Several studies conducted did not show any changes in FGF23 after exercise. ${ }^{15-17}$ One of which was the study conducted by Tarawan et al. (2019) that shows no changes in FGF23 gene expression in Wistar rat heart after short-term moderate-intensity exercise. ${ }^{17}$ This inconsistency was possibly due to different sample inclusion and training modalities given in the experiment.

Therefore, it is necessary to conduct a literature review that provides a comparative discussion about previous findings related to the effect of exercise on FGF23, especially serum
FGF23 which can reflect the systemic effect of exercise on FGF23. Besides, the exercise method will be described using the frequency, intensity, time, and type (FITT) formula. ${ }^{18}$

\section{METHOD}

This literature review is conducted in a qualitative study design. Previous studies related to the effect of exercise on serum FGF23 which were published in scientific journal articles for the last 10 years were collected, summarized in a comparative table, and analyzed in descriptive narration.

This literature review sorts scientific journal articles on Google Scholar and PubMed search engines. The keywords used in the article search are "FGF23", "exercise", "experimental study". These keywords are used separately or in combination. Only articles that are written in English or minimal have an English translation of the abstract (which contains information about research objectives, methods, results, and conclusion) would be selected. The selection of articles limited to the last 10 years of publication, were experimental study with exercise intervention in human or animal sample, and measured serum FGF23 or FGF23 protein expression in heart and/or skeletal muscle. The research flow is presented in figure 1.

The statement letter about denying any research misconduct such as plagiarism and data fabrication in the study was signed by the author on a legally accountable seal.

\section{RESULT}

The results of data collection related to the effect of exercise on FGF23 are presented in table 1.

From thirteen articles measuring serum FGF23 after exercise intervention, four articles were showing a significant increase in FGF23 after exercise. The four articles consist of three human subject studies (conducted by Lombardi et al., Emrich et al., and Kerschan-Schindl et al.) and one animal subject study conducted by $\mathrm{Li}$ et al. The other eight articles did not find any increase in FGF23 serum level after exercise consisted of five human subject studies and three animal subject studies. Only one human subject study, conducted by Neves et al., showed a decreased level of serum FGF23 after exercise intervention.

In the four studies that showed a significant increase in serum FGF23 after exercise, the exercise intervention tended to be more short term than studies that did not show changes in serum FGF23. The exercise interventions that showed positive results were carried out for one day to 3 weeks, while studies showing no change were carried out for six weeks to 12 months. However, one study by 
Emrich et al. in 2019 which investigated the acute effects of sympathetic activation on serum FGF23 did not show an increase in FGF23 after one-day exercise intervention.

The three human subject studies which showed increased FGF23 after exercise similarly conducted maximum intensity exercise. ${ }^{18}$ While the intensity of exercise in mice that showed positive results, was moderate. However, the relationship between intensity and the FGF23 serum concentration was still difficult to explain because most other studies that did not show changes in FG23 were also applied moderate to maximum intensity of exercise.

Time or duration of exercise in human subject studies that showed positive results was ranging from 5 hours exercise in a day to 88 hours total finishing time in the race. There are six human subject studies and four animal subject studies that applied exercise duration of up to one hour per day. Only one of the ten studies showed positive results regarding an increase in serum FGF23 after exercise.

Studies that showed an increase in human serum FGF23 were conducting cycling, hiking, and running exercise. While the studies in mice that showed positive results in FGF23 were conducting running (treadmills) exercise. Various other types of exercise such as aerobic variations (walking, jogging, cycling, etc.), strength variations (jumping quadriceps extensions, squats, biceps curls, pull-ups, etc.), balance variations (standing with feet together, on one leg, on balance board or planking) were also conducted in the studies but showed no increase in serum FGF23 and FGF23 protein expression.

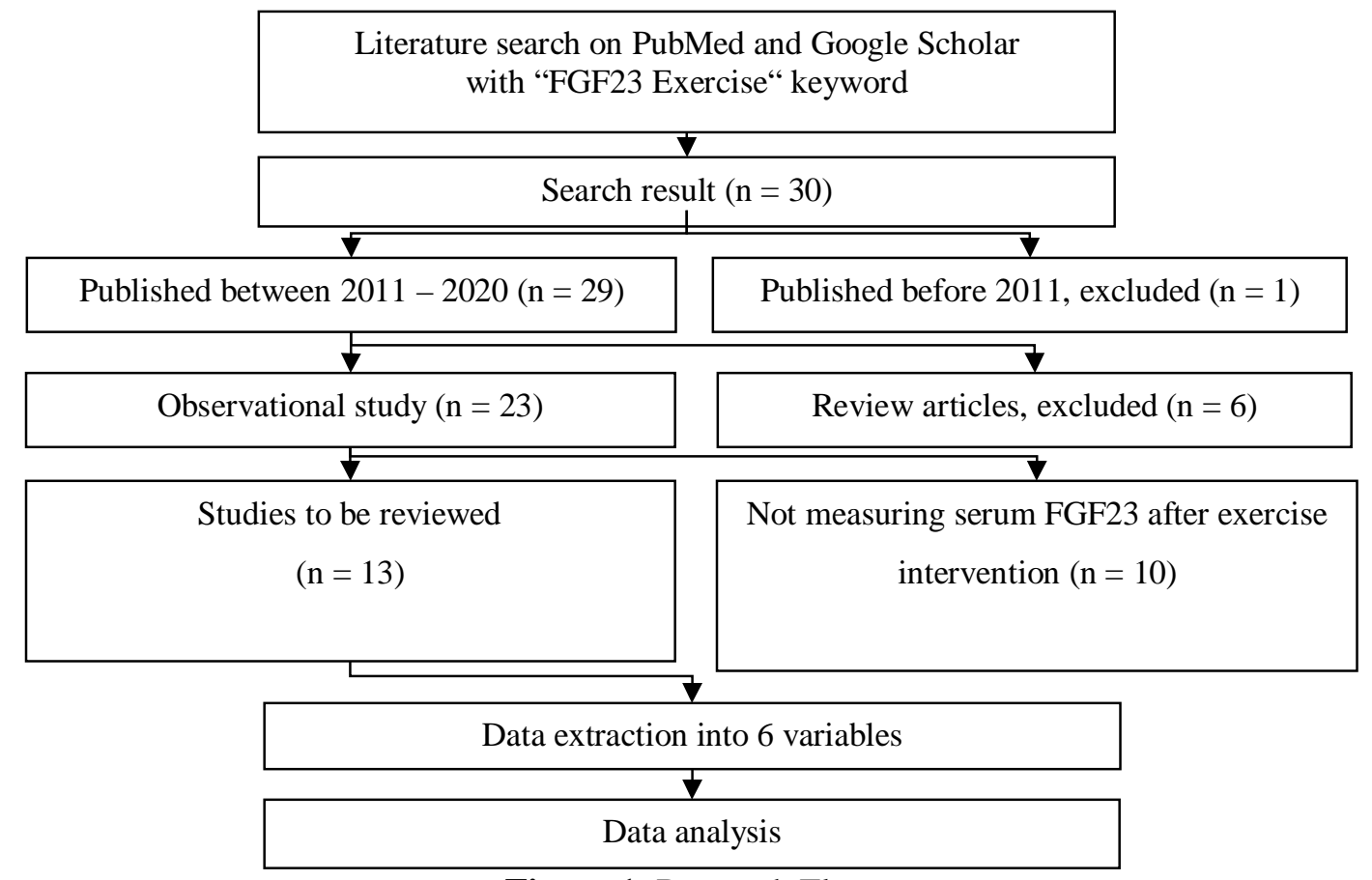

Figure 1. Research Flow

Table 1. Effect of Exercise on FGF23

\begin{tabular}{|c|c|c|c|c|c|c|}
\hline No & $\begin{array}{l}\text { Author } \\
\text { and Date }\end{array}$ & Purpose & Method & Sample & Findings & Conclusion \\
\hline 1. & $\begin{array}{l}\text { Lombardi } \\
\text { et al. } \\
2014^{12}\end{array}$ & $\begin{array}{l}\text { Determine the } \\
\text { effects of } \\
\text { endurance } \\
\text { exercise on the } \\
\text { bone-kidney- } \\
\text { parathyroid } \\
\text { hormonal axis } \\
\text { that regulates } \\
\text { calcium and } \\
\text { phosphorus } \\
\text { metabolism }\end{array}$ & $\begin{array}{l}\text { Giro d'Italia } 2011 \\
\text { race, } 3524.5 \mathrm{~km}, \\
\text { mean speed of } \\
\pm 36 \mathrm{~km} / \mathrm{h} \\
\text { F: } 21 \text { days' race ( } 2 \\
\text { days' rest) } \\
\text { I: - } \\
\text { T: Total finishing } \\
\text { times } 84 \mathrm{~h} 12 \mathrm{~m} 10 \mathrm{~s}- \\
88 \mathrm{~h} 15 \mathrm{~m} 51 \mathrm{~s} . \\
\text { T: Cycling }\end{array}$ & $\begin{array}{l}\text { Human } \\
\text { (Profession } \\
\text { al cyclists) } \\
\mathrm{I}_{\text {pre }}: 9 \\
\mathrm{I}_{\text {post }}: 9\end{array}$ & $\begin{array}{l}\text { Serum FGF23 on } \\
\text { day }-1=2.20 \pm \\
0.85 \mathrm{nmol} / \mathrm{L}, \\
2.76 \pm 0.71 \mathrm{nmol} / \mathrm{L} \\
\text { on day } 12 \text {, and } 3.37 \\
\pm 0.64 \mathrm{nmol} / \mathrm{L} \text { on } \\
\text { day } 22 \\
\uparrow 25(\mathrm{OH}) \mathrm{D} \text { (but not } \\
\text { significant), } \\
\uparrow \text { power output; } \\
\downarrow \text { BMI; Fat, serum } \\
\text { PTH, serum }\end{array}$ & $\begin{array}{l}\text { FGF23 was } \\
\text { increased by the } \\
\text { maximal } \\
\text { intensity } \\
\text { bicycle race }\end{array}$ \\
\hline
\end{tabular}




\begin{tabular}{|c|c|c|c|c|c|c|}
\hline No & $\begin{array}{c}\text { Author } \\
\text { and Date }\end{array}$ & Purpose & Method & Sample & Findings & Conclusion \\
\hline & & & & & $\begin{array}{l}\text { calcium were not } \\
\text { changed }\end{array}$ & \\
\hline 2. & $\begin{array}{l}\text { Wang et al. } \\
2015^{19}\end{array}$ & $\begin{array}{l}\text { Determine the } \\
\text { effects of } \\
\text { exercise on bone } \\
\text { and phosphate- } \\
\text { calcium } \\
\text { metabolism in } \\
\text { high-phosphorus } \\
\text { diet rat }\end{array}$ & $\begin{array}{l}\text { F: } 5 \mathrm{x} / \text { week, } 8 \\
\text { weeks } \\
\text { I: }- \\
\text { T: } 60 \text { seconds ( } 20 \mathrm{x} \\
\quad 3 \text { seconds) } \\
\text { T: Jumping (aerob) }\end{array}$ & $\begin{array}{l}\text { Rat } \\
\text { (Wistar, } \\
\text { male) } \\
\text { NC: } 10 \\
\text { NE: } 10 \\
\text { HC: } 11 \\
\text { HE: } 11\end{array}$ & $\begin{array}{l}\text { FGF23 serum level } \\
\text { was: } \\
\text { NC: } 411.8 \pm 53.1 \\
\text { pg/ml; NE: } 430.0 \pm \\
57.8 \mathrm{pg} / \mathrm{ml} \text {; HC: } \\
868.1 \pm 92.8 \mathrm{pg} / \mathrm{ml} \text {; } \\
\mathrm{HE}: 819.6 \pm 103.7 \\
\mathrm{pg} / \mathrm{ml} \\
\uparrow \text { serum inorganic } \\
\text { phosphorus, } 1-25 \\
\text { (OH) Vit D in high } \\
\text { phosphorus diet; } \\
\uparrow \text { serum total } \\
\text { calcium and } 1-25 \\
\text { (OH) Vit D in } \\
\text { exercise group; } \\
\uparrow \text { bone mineral } \\
\text { content, strength, } \\
\text { transverse and } \\
\text { longitudinal } \\
\text { thickness of the } \\
\text { tibial diaphysis. }\end{array}$ & $\begin{array}{l}\text { FGF23 was } \\
\text { increased by a } \\
\text { high } \\
\text { phosphorus diet } \\
\text { rather than by } \\
\text { exercise } \\
\text { intervention }\end{array}$ \\
\hline \multirow[t]{3}{*}{3.} & $\begin{array}{l}\text { Li et al. } \\
2016^{4}\end{array}$ & $\begin{array}{l}\text { Explore the role } \\
\text { FGF } 23 \text { in } \\
\text { exercise }\end{array}$ & $\begin{array}{l}\text { Acute exercise } \\
\mathrm{F}: 1 \mathrm{x} \\
\mathrm{I}: 20 \mathrm{~m} / \mathrm{min} \\
\quad \text { (moderate) } \\
\mathrm{T}: 60 \mathrm{~min} \\
\mathrm{~T}: \text { Running }\end{array}$ & $\begin{array}{l}\text { Mice } \\
\text { I }_{\text {short: }}: 8 \\
\text { I }_{\text {exhaustive }}: 8 \\
\text { I }_{\text {long: }} 8\end{array}$ & $\begin{array}{l}\uparrow \text { serum FGF23 in } \\
\text { every exercise } \\
\text { group, } \uparrow F G F 23 \\
\text { mRNA expression } \\
\text { in skeletal muscle } \\
\text { after chronic } \\
\text { exercise }\end{array}$ & $\begin{array}{l}\text { FGF23 was } \\
\text { increased after } \\
\text { short term, } \\
\text { exhaustive, and } \\
\text { long term } \\
\text { exercise }\end{array}$ \\
\hline & & & $\begin{array}{l}\text { Exhaustive exercise } \\
\mathrm{F}: 1 \mathrm{x} \\
\mathrm{I}: 20 \mathrm{~m} / \mathrm{min} \\
\quad \text { (moderate) } \\
\mathrm{T} \text { : Until exhaustion } \\
\mathrm{T} \text { : Running }\end{array}$ & & & \\
\hline & & & $\begin{array}{l}\text { Chronic exercise } \\
\text { F: } 1 \text { week } \\
\text { I: } 20 \mathrm{~m} / \mathrm{min} \\
\quad \text { (moderate) } \\
\text { T: } 60 \mathrm{~min} / \text { day } \\
\text { T: Running }\end{array}$ & & & \\
\hline
\end{tabular}




\begin{tabular}{|c|c|c|c|c|c|c|}
\hline No & $\begin{array}{c}\text { Author } \\
\text { and Date }\end{array}$ & Purpose & Method & Sample & Findings & Conclusion \\
\hline 4. & $\begin{array}{l}\text { Keshavarzi } \\
\text { et al. } 2017^{20}\end{array}$ & $\begin{array}{l}\text { Determine the } \\
\text { effect of aerobic } \\
\text { exercise on } \\
\text { FGF23, ACE, } \\
\text { and left } \\
\text { ventricular } \\
\text { hypertrophy }\end{array}$ & $\begin{array}{l}\text { F: 3x/week, } 12 \\
\text { weeks } \\
\text { I: 40-70\% HRmax } \\
\text { T: } 25-50 \mathrm{~min} \\
\text { T: Aerobic }\end{array}$ & $\begin{array}{l}\text { Human } \\
\text { (hyper- } \\
\text { tensive } \\
\text { elderly, } \\
\text { female) } \\
\text { I: } 10 \\
\text { C: } 10\end{array}$ & $\begin{array}{l}\text { FGF23 pre } \\
\text { exercise: } 340.92 \pm \\
59.29 \mathrm{pg} / \mathrm{ml} \text {; post } \\
\text { exercise: } 372.80 \pm \\
103.33 \mathrm{pg} / \mathrm{ml} ; p \\
\text { value between pre } \\
\text { and post test = } \\
0.43 \text {. } \\
\uparrow A C E \text { and } \uparrow l \text { left } \\
\text { ventricular } \\
\text { thickness after } \\
\text { aerobic exercise }\end{array}$ & $\begin{array}{l}\text { FGF23 was not } \\
\text { changed after } \\
\text { exercise }\end{array}$ \\
\hline
\end{tabular}

5. Emrich et al. $2018^{21}$ Emrich $e t$
al. $2019^{14}$

7. Liao et al. $2019^{16}$

$\begin{array}{ll}\begin{array}{l}\text { Evaluate the } \\ \text { effect of }\end{array} & \begin{array}{l}\text { Submaximal } \\ \text { exercise }\end{array} \\ \text { sympathetic } & \text { F: 1x } \\ \text { activation on } & \text { I: } 90 \% \text { IAT } \\ \text { FGF23 level } & \text { T: } 60 \text { min } \\ \text { T: static cycling } \\ \\ \\ \text { High-intensity } \\ \text { exercise } \\ \text { F: } 1 \mathrm{x} \\ \text { I: } 110 \% \text { IAT } \\ \text { T: until exhaustion } \\ \text { (<60 min) } \\ \text { T: static cycling }\end{array}$

Human Both groups: (exercise iFGF23 and cFGF23 regular-ly, were not increased, male) $\quad$ plasma phosphate, $\mathrm{I}_{\text {pre: }}: 15$ $\uparrow$ fraction excretion $\mathrm{I}_{\text {post }}: 15$ phosphate, $\downarrow$ fraction excretion calcium

个plasma calcium only in highintensity exercise.

$\begin{array}{lll}\text { Evaluate the } & \text { F: } 1 \mathrm{x} & \text { Human }(6 \\ \text { effect of } & \mathrm{I}:- & \text { females, } 2 \\ \text { increased } & \text { T: } 5 \text { hours } & \text { male) } \\ \begin{array}{l}\text { erythropoietin } \\ \text { (high-altitude }\end{array} & \text { T: hiking (at 2656m } & \mathrm{I}_{\text {pre }}: 8 \\ \text { above sea level) } & \mathrm{I}_{\text {post }}: 8\end{array}$

setting) on

FGF23 plasma

level in low and normal phosphorus diet
Determine the effect of exercise on bone mineralization in CKD patient
F: 5x/week, 8
weeks
I: $(9-16 \mathrm{~m} / \mathrm{min})$
T: $10-60 \mathrm{~min}$
$\mathrm{T}$ : running

cFGF23 had a single peak after 5 hours high-altitude hike in the normal (92.3 $\mathrm{RU} / \mathrm{ml}$ ) and low phosphorus diet group (187.6 $\mathrm{RU} / \mathrm{ml}$ )

$\uparrow \mathrm{EPO}, \downarrow \mathrm{PTH}$,

$\downarrow$ urinary phosphorus excretion, $\uparrow$ urinary calcium excretion

Rats (Sprague Dawley, CKD, male)

I: 13

C: 9

$\mathrm{C}_{\text {nonCKD }}: 8$
FGF23 levels in the rats from the CKD + exercise group was $723.9 \pm 57.1 \mathrm{pg} / \mathrm{mL}$, in the CKD group (non exercise) was $836.1 \pm 71.4 \mathrm{pg} / \mathrm{mL}$, in the control (nonCKD and non exercise) was 404.6 $\pm 64.8 \mathrm{pg} / \mathrm{mL}$
FGF23 was not changed after submaximal nor high-intensity exercise
cFGF23 (C fragment FGF23, but not iFGF23 (intact FGF23), had increased after 5 hours exercise in high altitude
Increased FGF23 was markedly determined by CKD condition rather than exercise intervention. 


\begin{tabular}{|c|c|c|c|c|c|c|}
\hline No & $\begin{array}{c}\text { Author } \\
\text { and Date }\end{array}$ & Purpose & Method & Sample & Findings & Conclusion \\
\hline & & & & & $\begin{array}{l}\downarrow_{\text {serum }} \text { sclerostin, } \\
\downarrow \text { CTX-1 } \\
\text { resorption marker), } \\
\uparrow \text { tibial } \beta \text {-catenin in } \\
\text { the Intervention } \\
\text { group. }\end{array}$ & \\
\hline & & & & & $\begin{array}{l}\text { Serum intact } \\
\text { parathyroid } \\
\text { hormone, alkaline } \\
\text { phosphatase, } \\
\text { calcium, and } \\
\text { phosphate were not } \\
\text { changed }\end{array}$ & \\
\hline
\end{tabular}

8. Buskermol Investigate the Peak power training en et al. $2019^{15}$

(PT)

F: 5x/week, 6

effect of
different training
modalities on
FGF23 and $1 \alpha-$
hydroxylase
(CYP27b1) in rat
bone

weeks

I: Maximal

$\mathrm{T}: 15 \mathrm{~s}$ in gallop

$\mathrm{T}: 10$ sprints

(anaerob)

\section{Endurance training}

(ET)

F: $5 x /$ week, 6 weeks

I: $16-26 \mathrm{~m} / \mathrm{min}$

$\mathrm{T}: 10-45 \mathrm{~min}$

$\mathrm{T}$ : Endurance

(running on a treadmill)

Combined training (PET

Conducting PT and ET with 8 hours' interval

\section{Endurance and} $2020^{13,22}$

\section{balance exercise}

Investigate the effect of exercise on abdominal aortic calcification in CKD patients combination (EB)

F: 3-5x/week, 12 months

I: $13-17 \mathrm{RPE}$

$\mathrm{T}: 150 \mathrm{~min} /$ week (60 min endurance +90

$\mathrm{T}$ min balance)

- Endurance: Aerobic variation

- Balance: planking, step on one foot, etc.
Rat

(Wistar)

$\mathrm{I}_{\mathrm{PT}}: 6$

$\mathrm{I}_{\mathrm{ET}}: 6$

$\mathrm{I}_{\mathrm{PET}}: 9$

C: 7

There was no

significant difference in FGF23 serum concentration in PT, ET, nor PET to control group

Lower sclerostin (SOST), dental matrix protein 1 (DMP1), Phosphate Regulating Endopeptidase Homolog X-Linked (PHEX), matrix extracellular phosphoglycoprotei n (MEPE), CYP27b1, and vitamin D receptor (VDR) in PT group

Higher Serum PTH concentrations in the PT group

\begin{tabular}{|c|c|c|}
\hline $\begin{array}{l}\text { Human } \\
\text { (CKD) } \\
\text { I }_{E B}: 59 \\
\text { IES }_{53}\end{array}$ & $\begin{array}{l}\text { the p-value for } \\
\text { plasma FGF } 23 \\
\text { concentration in the } \\
\text { EB group was } 0.4 \text {, } \\
\text { in the ES group was } \\
0.4 \text {, and in the } \\
\text { whole group was } 0.2 \\
\downarrow \text { mGFR, } \\
\downarrow \text { lipoprotein, in both } \\
\text { groups. } \\
\uparrow P T H, \\
\uparrow 1,25(\mathrm{OH})_{2} \mathrm{D} 3 \\
\text { increased in the } \\
\text { strength group } \\
\uparrow \text { fetuin-A increased }\end{array}$ & $\begin{array}{l}\text { FGF23 was not } \\
\text { changed after } \\
12 \text { months of } \\
\text { exercise in } \\
\text { CKD patients }\end{array}$ \\
\hline
\end{tabular}

FGF23 was not changed after all training modalities after six weeks of exercise 


\begin{tabular}{|c|c|c|c|c|c|c|}
\hline No & $\begin{array}{c}\text { Author } \\
\text { and Date }\end{array}$ & Purpose & Method & Sample & Findings & Conclusion \\
\hline & & & $\begin{array}{l}\text { Endurance and } \\
\text { strength exercise } \\
\text { combination }(E S) \\
\mathrm{F}: \text { 3-5x/week, } 12 \\
\text { months } \\
\mathrm{I}: \text { 13-17 RPE } \\
\mathrm{T}: \text { 150min/week } \\
\quad(60 \mathrm{~min} \\
\text { endurance + } 90 \\
\text { min strength) } \\
\mathrm{T} \quad \\
-\quad \text { Endurance: } \\
\text { aerobic variation } \\
\text { - } \text { Strength: } \\
\text { quadriceps } \\
\text { extension, squats, } \\
\text { biceps curls, } \\
\text { pull-ups, etc. }\end{array}$ & & $\begin{array}{l}\text { in the balance group } \\
\text { Plasma } \\
\text { triglycerides, total } \\
\text { cholesterol, high- } \\
\text { density lipoprotein } \\
\text { cholesterol, low- } \\
\text { density lipoprotein } \\
\text { cholesterol, FGF23, } \\
\text { phosphate, calcium, } \\
\text { IL6, C-reactive } \\
\text { protein (CRP), } \\
\text { albumin were } \\
\text { unchanged }\end{array}$ & \\
\hline 10. & $\begin{array}{l}\text { Fakhrpour } \\
\text { et al. } \\
2020^{23}\end{array}$ & $\begin{array}{l}\text { Investigate the } \\
\text { efficacy of } \\
\text { aerobic and } \\
\text { resistance } \\
\text { exercise } \\
\text { combination on } \\
\text { quality of life } \\
\text { and calcification } \\
\text { markers } \\
\text { including FGF23 } \\
\text { in hemodialysis } \\
\text { patients }\end{array}$ & $\begin{array}{c}\text { F: } 3 x / \text { week, } 4 \\
\text { months } \\
\text { I: } 12-14 \text { RPE for } \\
\text { aerobic exercise, } \\
12 \text { repetitions (1- } \\
3 \text { sets) for } \\
\text { resistance } \\
\text { exercise } \\
\mathrm{T}: 10-45 \text { min } \\
\mathrm{T}: \text { Combined } \\
\text { aerobic (static } \\
\text { cycling) and } \\
\text { resistance } \\
\text { (foreleg } \\
\text { resistance) }\end{array}$ & $\begin{array}{l}\text { Human, } \\
\text { (Hemodial } \\
\text { ysis } \\
\text { patient, } 38 \\
\text { male, } 7 \\
\text { female) I: } \\
24 \\
\text { C: } 21\end{array}$ & $\begin{array}{l}\text { FGF23 in the } \\
\text { exercise group was } \\
273.11 \pm 82.28 \\
\text { before exercise and } \\
294.67 \pm 88.11 \text { after } \\
\text { exercise. } \\
\text { p-value was }>0.05 \\
\text { compared to control } \\
\uparrow \text { klotho, } \\
\downarrow \text { phosphorus, } \\
\downarrow \text { PTH, } \\
\text { fetuin-A was not } \\
\text { changed }\end{array}$ & $\begin{array}{l}\text { FGF23 was not } \\
\text { changed after } \\
16 \text { weeks of } \\
\text { exercise } \\
\text { intervention }\end{array}$ \\
\hline 11. & $\begin{array}{l}\text { Ramanjan- } \\
\text { eya et al. } \\
2020^{24}\end{array}$ & $\begin{array}{l}\text { Investigate the } \\
\text { response of } \\
\text { FGF19 } \\
\text { subfamily and } \\
\text { fetuin-A to an } \\
\text { acute intralipid, } \\
\text { insulin infusion } \\
\text { and exercise }\end{array}$ & $\begin{array}{c}\mathrm{F}: 3 \mathrm{x} / \text { week, } 8 \\
\text { weeks } \\
\mathrm{I}: 60 \% \mathrm{VO}_{2} \mathrm{max} \\
\quad \text { (moderate) } \\
\mathrm{T}: 60 \mathrm{~min} \\
\mathrm{~T}: \text { walking } \\
\quad \text { (treadmill) }\end{array}$ & $\begin{array}{l}\text { Human, } \\
\text { (PCOS, } \\
\text { female) } \\
\text { IPCOS: } 11 \\
\text { Ihealthy: } 10\end{array}$ & $\begin{array}{l}\text { FGF23, FGF19, } \\
\text { FGF21, fetuin-A } \\
\text { were not changed } \\
\text { after exercise in } \\
\text { PCOS and healthy } \\
\text { group }\end{array}$ & $\begin{array}{l}\text { FGF23 was not } \\
\text { changed after } 8 \\
\text { weeks of } \\
\text { exercise } \\
\text { intervention }\end{array}$ \\
\hline 12. & $\begin{array}{l}\text { Neves et al. } \\
2020^{25}\end{array}$ & $\begin{array}{l}\text { Compare the } \\
\text { effectiveness of } \\
\text { dynamic and } \\
\text { isometric } \\
\text { resistance } \\
\text { training in } \\
\text { osteogenesis of } \\
\text { maintenance } \\
\text { hemodialysis } \\
\text { patients }\end{array}$ & $\begin{array}{l}\text { Dynamic resistance } \\
\text { training }(D R T) \\
\mathrm{F}: 3 \mathrm{x} / \mathrm{week}, 6 \\
\text { months } \\
\text { I: low to moderate } \\
\quad \text { intensity } \\
\text { T: } 8-12 \text { repetitions, } \\
3 \text { sets, time under } \\
\text { tension each set } \\
\text { was } 32-48 \mathrm{~s} \\
\text { T: Resistance training } \\
\\
\text { Isometric resistance } \\
\text { training (IRT) }\end{array}$ & $\begin{array}{l}\text { Human } \\
\text { (Hemodial } \\
\text { ysis } \\
\text { patient) } \\
\text { I }_{\text {DRT: } 66} \\
\text { I }_{\text {IRT }} 67 \\
\text { C: } 60\end{array}$ & $\begin{array}{l}\text { Lower FGF23 after } \\
\text { exercise in DRT } \\
\text { group (compared to } \\
\text { baseline/before } \\
\text { exercise, control, } \\
\text { and IRT group) } \\
\text { DRT: } \uparrow \text { klotho, } \\
\uparrow 1,25(\mathrm{OH})_{2} \mathrm{D}, \\
\downarrow \text { phosphate, } \\
\downarrow \text { calcium phosphate, } \\
\downarrow \text { PTH } \\
\text { IRT: } \uparrow \text { klotho, }\end{array}$ & $\begin{array}{l}\text { FGF23 was } \\
\text { decreased after } \\
6 \text { months of } \\
\text { dynamic } \\
\text { resistance } \\
\text { training in } \\
\text { maintenance } \\
\text { hemodialysis } \\
\text { patients }\end{array}$ \\
\hline
\end{tabular}




\begin{tabular}{|c|c|c|c|c|c|c|}
\hline No & $\begin{array}{c}\text { Author } \\
\text { and Date }\end{array}$ & Purpose & Method & Sample & Findings & Conclusion \\
\hline & & & $\begin{array}{l}\text { Same as DRT but } \\
\text { performed isometric } \\
\text { contractions in each } \\
\text { type of exercise }\end{array}$ & & $\begin{array}{l}\uparrow 1,25(\mathrm{OH})_{2} \mathrm{D}, \\
\downarrow \text { calcium } \\
\text { phosphate, } \downarrow \text { PTH }\end{array}$ & \\
\hline
\end{tabular}

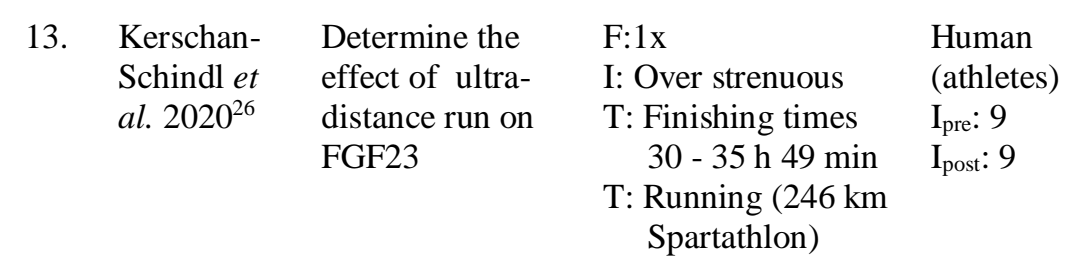

\author{
$\uparrow \mathrm{FGF} 23$ \\ (from $2.2 \mathrm{pmol} / \mathrm{L}$ \\ before race to 14.4 \\ pmol/L 15 minutes \\ after race, $\mathrm{p}=$ \\ 0.001). But then fell \\ to $1.4 \mathrm{pmol} / \mathrm{L}$ on the \\ three days after the \\ start of the race $(\mathrm{p}<$ \\ 0.0001)
}

\author{
FGF23 was \\ increased after \\ short term \\ maximal \\ intensity \\ exercise
}

Phosphate and blood urea nitrogen were not changed

\section{DISCUSSION}

\section{Increased FGF23 Expression in Blood Serum}

Four out of thirteen studies measuring serum FGF23 after exercise intervention were showed a significant increase in serum FGF23 after exercise. $^{4,12,14,26}$ In the four studies, the exercise intervention tended to be more short term than studies that did not show changes in serum FGF23. The exercise intervention that showed positive results ranged from one day to three weeks, while studies showing no change were carried out for six weeks to 12 months. ${ }^{13,15,20,23-25}$ The absence of an increase in serum FGF23 after long term exercise was probably due to metabolic adaptation in the study subjects. ${ }^{15}$ After repeated exposure to exercise, the body made adaptations to conserve energy which causes the body to become more 'blunt' to exercise. ${ }^{2}$ One of the consequences of this metabolic adaptation was reduced inflammation reaction in exercise, which is one of the stimulators of FGF23 production. ${ }^{27}$ This presumption is supported by the study conducted by Rosa et al. 2020 which found that inflammation and basal FGF23 were lower in master athletes. ${ }^{28}$

One of the studies which showed an increase in serum FGF23 was conducted by Lombardi et al. $2014 .{ }^{12}$ Nine professional cyclists were recruited in the study and showed an increase in serum FGF23 after three weeks of a cycling race. The blood sampling was carried out three times throughout the race, one day before the race, the $12^{\text {th }}$ day, and the $22^{\text {nd }}$ day. The increase of serum FGF23 was significant between the day before the race and the $22^{\text {nd }}$ day. ${ }^{12}$ However, the increase of serum FGF23 in these regularly exercised subject could also be determined by another factor besides the exercise intervention, that is the high phosphorus diet which will be discussed further in the next section.

The participants in the study conducted by Lombardi et al. 2014 took approximately 84 to 88 hours in 21 days (with 2 days for rest) to complete $3524.5 \mathrm{~km}$ of the stage. Thus it could be assumed that the participants performed the maximumintensity exercise with a duration of 4 to 5 hours per day. It turns out that the intensity and duration of this exercise are similar to the conditions that caused an increase in serum FGF23 in the study by Emrich et al. 2019. ${ }^{14}$ In that study, serum FGF23 was only found to be increased after 5 hours of maximal intensity hiking and back to average result in the next blood sampling time point which was 13 hours later. One recent study by Kerschan-Schindl et al. 2020 also found an increase in serum FGF23 in nine athletes after finishing $246 \mathrm{~km}$ Spartathlon for 30 to 35 hours. $^{26}$

Those studies suggested that the intensity and time or duration of exercise which could increase human serum FGF23 was generally a maximal intensity with long-duration exercise. ${ }^{12,14,26}$ The long-duration maximal intensity exercise possibly has caused sufficient release of myokine IL-6, inflammatory response, and hypoxic state to stimulate increased production of FGF23 in the bone which is further released in the bloodstream. ${ }^{27}$ 
However, those studies have other factors that could be another stimulator for FGF23 production such as high phosphorus diet and increased erythropoietin at high altitudes. Therefore, it could not be concluded whether long-duration maximum intensity exercise alone was sufficient to increase FGF23. ${ }^{27}$

In contrast to those studies, the study conducted by Emrich et al. 2018 which measured the effect of sympathetic activation after exercise was found no increase in serum FGF23 even after shortterm exercise. Serum FGF23 was measured in the study after a single submaximal or high-intensity exercise that was conducted for up to 60 minutes. ${ }^{21}$ The subjects of this study were healthy men which exercised regularly, thus the metabolic adaptation probably occurred before the exercise intervention in this study. This metabolic adaptation might cause the 60 minutes maximal intensity exercise became insufficient to increase serum FGF23 in the study subjects. Moreover, there were no other additional factors in this study that could increase FGF23 such as high phosphorus and erythropoietin such as in Lombardi et al. 2014 and Emrich et al. 2019 studies. ${ }^{12,14}$

Regarding the type of exercise, studies that showed an increase in serum FGF23 were conducted in several types of exercise. Studies that showed an increase in serum FGF23 applied exercise types of hiking, cycling, and running., ${ }^{4,14}$ However, other studies with longer exercise intervention (six weeks to 12 months) also applied running and cycling type of exercise but did not show an increase in serum FGF23. ${ }^{13,15,16,23}$ Furthermore, a study by Zhou et al. 2020 which applied a different combination of endurance, balance, and strength types also found no increase in serum FGF23 in every group after 12 weeks of exercise intervention. ${ }^{13}$ Therefore, serum FGF23 is more likely to be influenced by exercise duration than by type of exercise.

In animal studies, Li et al. (2016) were found that serum FGF23 increased in the exercise carried out for one day to one week, moderate CONCLUSION

Serum FGF23 was found to be increased after one day to three weeks of exercise. Serum intensity, duration of up to 60 minutes, and the type of exercise was running on a treadmill ${ }^{4}$. However, this result could not be compared to other animal studies that also measured serum FGF23 after exercise because the other studies used long-term exercise intervention (six to eight weeks) which is a crucial factor that largely determines the serum FGF23 findings as discussed earlier. ${ }^{15,16,19}$ Therefore it was difficult to conclude which intensity, time, and type of exercise could increase serum FGF23 in animal studies.

\section{Supplementation}

As the main physiological function of FGF23 is to regulate phosphorus and vitamin D, FGF23 regulation is very closely related to bone mineralization ${ }^{7}$. Phosphate, calcium, vitamin $\mathrm{D}$, and PTH levels in the blood are closely related to serum FGF23. ${ }^{29}$ In the study of Lombardi et al., there was high dietary phosphorus intake, $3874.97 \mathrm{mg} /$ day, which could be the main factor in increasing serum FGF23 in the study. ${ }^{12}$ The high phosphorus diet could be one major confounder in the study, especially supported by Wang et al. findings which showed that serum FGF23 was increased by a high phosphorus diet rather than by the given exercise intervention. ${ }^{19}$

\section{Recommendations}

A further study which aimed to elucidate the direct physiologic effect of exercise on FGF23 level in serum and skeletal muscle need to consider including untrained individual, short term maximal intensity exercise, and restrict several diet component related to bone mineral regulators, such as phosphorus, to minimize confounding factors.

Another biomolecular investigation needs to be done to elucidate the mechanism of FGF23 related to exercise performance, also to distinguish the physiologic and pathologic levels of FGF23.

FGF23 in human studies found to be increased after long-duration maximal-intensity exercise.

\section{FUNDING} sources.

This research was not funded by any grant

\section{CONFLICT OF INTEREST}

This research has no conflict of interest. 


\section{REFERENCES}

1. WHO | Physical Activity [Internet]. [cited 2020 Jun 29]. Available from: https://www.who.int/dietphysicalactivity/pa/en/

2. Pontzer H. Energy constraint as a novel mechanism linking exercise and health. Physiology. 2018;33(6):384-93.

3. Paley CA, Johnson MI. Abdominal obesity and metabolic syndrome: Exercise as medicine? BMC Sports Sci Med Rehabil. 2018;10(1):1-8.

4. Li DJ, Fu H, Zhao T, Ni M, Shen FM. Exercisestimulated FGF23 promotes exercise performance via controlling the excess reactive oxygen species production and enhancing mitochondrial function in skeletal muscle. Metabolism. 2016;65(5):747-56.

5. Dolegowska K, Marchelek-Mysliwiec M, Nowosiad-Magda M, Slawinski M, Dolegowska B. FGF19 subfamily members: FGF19 and FGF21. J Physiol Biochem. 2019;75(2):229-40.

6. Li X. The FGF metabolic axis. Front Med. 2019;13(5):511-30.

7. Kovesdy CP, Quarles LD. FGF23 from bench to bedside. Am J Physiol - Ren Physiol. 2016;310(11):F1168-74.

8. Hanks LJ, Casazza K, Judd SE, Jenny NS, Gutiérrez OM. Associations of fibroblast growth factor-23 with markers of inflammation, insulin resistance and obesity in adults. PLoS One. 2015;10(3):1-12.

9. Avin KG, Vallejo JA, Chen NX, Wang K, Touchberry CD, Brotto M, et al. Fibroblast growth factor 23 does not directly influence skeletal muscle cell proliferation and differentiation or ex vivo muscle contractility. Am J Physiol - Endocrinol Metab. 2018;315(4):E594-604.

10. Richter B, Faul C. FGF23 actions on target tissues-with and without Klotho. Vol. 9, Frontiers in Endocrinology. Frontiers Media S.A.; 2018. p. 189.

11. David V, Francis C, Babitt JL. Ironing out the cross talk between FGF23 and inflammation. Am J Physiol - Ren Physiol. 2017;312(1):F1-8.

12. Lombardi G, Corsetti R, Lanteri P, Grasso D, Vianello E, Marazzi MG, et al. Reciprocal regulation of calcium-/phosphate-regulating hormones in cyclists during the Giro d'Italia 3week stage race. Scand J Med Sci Sport. 2014;24(5):779-87.

13. Zhou Y, Hellberg M, Hellmark T, Höglund P, Clyne N. Twelve months of exercise training did not halt abdominal aortic calcification in patients with CKD - a sub-study of RENEXC-a randomized controlled trial. BMC Nephrol.

\section{0;21(1):233.}

14. Emrich IE, Dederer J, Kircher A, Klemis V, Lennartz CS, Untersteller K, et al. Does a rise in plasma erythropoietin after high-altitude exposure affect FGF23 in healthy volunteers on a normal or low-phosphorus diet? Nutr Metab Cardiovasc Dis. 2019;29(12):1361-7.

15. Buskermolen J, Van Der Meijden K, Furrer R, Mons DJ, Van Essen HW, Heijboer AC, et al. Effects of different training modalities on phosphate homeostasis and local Vitamin D metabolism in rat bone. PeerJ. 2019;2019(1):117.

16. Liao HW, Huang TH, Chang YH, Liou HH, Chou YH, Sue YM, et al. Exercise alleviates osteoporosis in rats with mild chronic kidney disease by decreasing sclerostin production. Int J Mol Sci. 2019;20(8).

17. Tarawan VM, Gunadi JW, Surbakti TAB, Widowati W, Goenawan H, Lesmana R. Effect of Acute Physical Exercise with Moderate Intensities on FGF23 Gene Expression in Wistar Rat Heart. Maj Kedokt Bandung. 2019 Dec 21;51(4):221-5.

18. Riebe D, Ehrman JK, Liguori G, Magal M, editors. ACSM's Guidelines for Exercise Testing and Prescription. tenth. Vol. 35, American College of Sports Medicine. Philadelphia, PA: Wolters Kluwer Health; 2018. 35-6295-35-6295 p.

19. Wang G, Honda A, Mizuno T, Harada K, Sogo $\mathrm{N}$, Umemura Y. Effects of high phosphorous intake and jump training on the developing rat tibia. Japanese J Phys Fit Sport Med. 2015;64(3):295-303.

20. Keshavarzi Z, Daryanoosh F, Jahromi MK, Mohammadi M. The effect of 12 weeks of aerobic exercise on plasma levels of fibroblast growth factor 23, Angiotensin converting enzyme and left ventricular hypertrophy in hypertensive elderly women. J Shahid Sadoughi Univ Med Sci. 2017;25(3):222-9.

21. Emrich IE, Baier M, Zawada AM, Meyer T, Fliser D, Scharhag J, et al. Plasma FGF23 does not rise during physical exercise as a physiological model of sympathetic activation. Clin Res Cardiol. 2018;108(3):341-3.

22. Hellberg M, Höglund P, Svensson P, Clyne N. Randomized Controlled Trial of Exercise in CKD-The RENEXC Study. Kidney Int Reports. 2019;4(7):963-76.

23. Fakhrpour R, Khosroshahi HT, Ebrahim K, Ahmadizad S, Abbasnejad M, Abbasi MM, et al. Effect of sixteen weeks combined training on FGF-23, Klotho, and Fetuin-A levels in 
patients on maintenance hemodialysis. Iran $\mathbf{J}$ Kidney Dis. 2020;14(3):212-8.

24. Ramanjaneya M, Bensila M, Bettahi I, Jerobin J. Dynamic Changes in Circulating Endocrine FGF19 Subfamily and Fetuin-A in Response to Intralipid and Insulin Infusions in Healthy and PCOS Women. Front Endocrinol (Lausanne). 2020;11(September):1-10.

25. Neves RVP, Corrêa HL, Deus LA, Reis AL, Michel K, Simões HG, et al. Dynamic not isometric training blunts osteo-renal disease by the sclerostin/FGF23/Klotho axis in maintenance hemodialysis patients: a randomized clinical trial. J Appl Physiol. 2020;55(61).

26. Kerschan-schindl K, Skenderi K, Wahl-figlash
K, Gelles K, Föger-samwald U, Thalmann M, et al. Increased serum levels of fibroblast growth factor 23 after an ultradistance run. J Sci Med Sport. 2020;

27. Bär L, Stournaras C, Lang F, Föller M. Regulation of fibroblast growth factor 23 (FGF23) in health and disease. FEBS Lett. 2019;593(15):1879-900.

28. Rosa TS, Neves RVP, Deus LA, Sousa CV, da Silva Aguiar S, de Souza MK, et al. Sprint and endurance training in relation to redox and inflammatory status and biomarkers of aging in master athletes. Nitric Oxide. 2020;

29. Fukumoto S. Fibroblast growth factor 23. Principles of Bone Biology. Elsevier Inc.; 2019. 1529-1538 p. 